\title{
ON THE STABILITY OF CAVITATING EQUILIBRIA
}

\author{
BY

\section{J. SIVALOGANATHAN}

School of Mathematical Sciences, University of Bath, Claverton Down, Bath, UK

1. On the stability of cavitating equilibria. The mathematical phenomenon of cavitation in the setting of finite elasticity was first demonstrated by Ball in [3]. This work was subsequently extended and generalised in $[23,21,15]$ and many aspects of the problem are now well understood (see $[1,9,10,11,12,15,17,22,18,8]$ and the references therein). It is known that these cavitating equilibria, which correspond to discontinuous radial deformations of a ball of hyperelastic material, are often global minimisers of the stored energy in classes of radial maps of the ball (see [3, 21, 22]). In this paper we demonstrate certain stability properties of these cavitating equilibria with respect to general (not necessarily radial) variations in $W^{1, P}(B)$.

The setting for this work is a ball of compressible isotropic hyperelastic material occupying the region $B=\left\{\mathbf{x} \in \mathbf{R}^{3}:|\mathbf{x}|<1\right\}$ in its reference state. We refer to maps $\mathbf{u}: \bar{B} \rightarrow \mathbf{R}^{3}$ as deformations of the ball and we seek equilibria by minimising the corresponding stored energy

$$
E(\mathbf{u})=\int_{B} W(\nabla \mathbf{u}(\mathbf{x})) d x
$$

over admissible deformations. (Here $W: M_{+}^{3 \times 3} \rightarrow \mathbf{R}^{+}$is the stored energy function of the material and $M_{+}^{3 \times 3}$ denotes the space of real $3 \times 3$ matrices with positive determinant.) The equilibrium equations are the Euler-Lagrange equations for $E$, namely

$$
\frac{\partial}{\partial x^{\alpha}}\left[\frac{\partial W}{\partial F_{\alpha}^{i}}(\nabla \mathbf{u}(\mathbf{x}))\right]=0 \quad \text { for } \mathbf{x} \in B, i=1,2,3 .
$$

In the displacement boundary-value problem the ball is held under a prescribed radial boundary condition $\mathbf{u}(\mathbf{x})=\lambda \mathbf{x}$ for $\mathbf{x} \in \partial B$ (where $\lambda>0$ is a constant). It is known that, under suitable hypotheses on $W$ (see $[3,21]$ ), there is a critical value $\lambda_{\text {crit }}$ such that for all $\lambda \leq \lambda_{\text {crit }}$ the homogeneous deformation

$$
\mathbf{u}^{\text {hom }}(\mathbf{x}) \equiv \lambda \mathbf{x}
$$

is the global minimiser of $E$ in the class of radial deformations of $B$. However, for each $\lambda>\lambda_{\text {crit }}$ there is a unique (cavitating) map $\mathbf{u}_{c(\lambda)}(\mathbf{x})$ which produces a 
spherical cavity of radius $c(\lambda)$ at the centre of the ball which is the global radial minimiser. Moreover, it is known that $\mathbf{u}_{c(\lambda)}$ is a weak solution of the corresponding, three-dimensional equations (see [3]) and that the radius $c(\lambda)$ of the cavity produced is a monotone increasing function of $\lambda$, with $c(\lambda) \searrow 0$ as $\lambda \searrow \lambda_{\text {crit }}$ (see, e.g., [3, 21]).

In Theorem 2.5, under suitable hypotheses on $W$, we prove the following stability property for the cavitating equilibrium close to initiation: given any $\delta \in(0,1)$ and any tolerance $M>0$, if $c(\lambda)$ is sufficiently small, then

$$
E(\mathbf{u}) \geq E\left(\mathbf{u}_{c}\right) \text { for all } \mathbf{u}-\mathbf{u}_{c}=\phi \in W_{0}^{1, P}\left(B / \bar{B}_{\delta}\right), P>3,
$$

with $\left\|\mathbf{u}-\mathbf{u}_{c}\right\|_{\infty} \leq M$, where $B_{\delta}=\left\{\mathbf{x} \in \mathbf{R}^{3}:|\mathbf{x}|<\delta\right\}$ and $\|\cdot\|_{\infty}$ denotes the $L^{\infty}$ norm. Notice that the admissible deformations $\mathbf{u}$ appearing in (1.4) only differ from $\mathbf{u}_{c}$ away from the cavity; moreover, by the Sobolev embedding Theorem, $\phi$ may be taken as continuous, and so the deformation $\mathbf{u}=\mathbf{u}_{c}+\phi$ does not change the shape of the cavity nor does it introduce further holes. Hence the cavitating equilibrium is a strong local minimiser in any $L^{\infty}$ neighbourhood, sufficiently close to initiation of cavitation, with respect to these continuous variations supported outside $B_{\delta}$.

We also give an alternative stability result in Corollary 2.6 (which follows from the proof of Theorem 2.5) in which $\delta$ is not fixed but depends on $c$ : this shows that, for small cavity sizes $c, \mathbf{u}_{c}$ is stable with respect to perturbations $\phi$ supported outside a boundary layer proportional to $c$ provided that $\|\phi\|_{\infty}$ is of the order of the thickness of the layer.

These results allowing continuous perturbations contrast with the recent interesting work of James and Spector [12] which shows, that for certain stored energy functions, the energy of a cavitating equilibrium $\mathbf{u}_{c(\lambda)}$ can always be reduced by introducing line discontinuities. Interestingly, the uniformly polyconvex stored energies we consider do not satisfy the hypotheses of [12].

In Sec. 3 we consider variations $\mathbf{u}-\mathbf{u}_{c}=\phi \in W_{0}^{1, P}(B)$ for $P<3$ and show that the cavitating equilibria are not strict local minimisers of the energy in $L^{\infty}(B) \cap$ $W^{1, P}(B)$ : in particular we show that given any $\varepsilon>0$ there exists $\mathbf{u} \in W^{1, P}(B)$, $\left\|\mathbf{u}-\mathbf{u}_{c}\right\|_{\infty}<\varepsilon$, with $E(\mathbf{u})=E\left(\mathbf{u}_{c}\right)$.

In Sec. 4 we prove stability of cavitating equilibria with respect to a class of variations which permits limited variations in the cavity shape.

We next outline the idea behind our main proof of stability. We will make extensive use of the notion of polyconvexity: recall that a stored energy function $W$ is said to be polyconvex if

$$
W(F)=G(F, \operatorname{Adj} F, \operatorname{det} F) \text { for all } F \in M^{3 \times 3},
$$

where $\operatorname{Adj} F$ denotes the transposed matrix of cofactors of $F$ and

$$
G: M^{3 \times 3} \times M^{3 \times 3} \times \mathbf{R} \rightarrow \mathbf{R}^{+} \cup\{\infty\}
$$

is convex (see $[2,7])$.

We assume throughout this paper that $W$ is a uniformly polyconvex stored energy function in the sense that there exists $\kappa>0$ such that

$$
W(F)=\kappa|F|^{2}=\widetilde{W}(F) \text { for all } F \in M^{3 \times 3},
$$


where $\widetilde{W}$ is polyconvex. Thus, by the polyconvexity of $\widetilde{W}$, we have

$$
\begin{aligned}
E(\mathbf{u}) \geq & \int_{B} \kappa|\nabla \mathbf{u}|^{2}+\widetilde{G}\left(\nabla \mathbf{u}_{c}, \operatorname{Adj} \nabla \mathbf{u}_{c}, \operatorname{det} \nabla \mathbf{u}_{c}\right) \\
& +\frac{\partial \widetilde{G}}{\partial F_{\alpha}^{i}}\left(\nabla \mathbf{u}_{c}, \operatorname{Adj} \nabla \mathbf{u}_{c}, \operatorname{det} \nabla \mathbf{u}_{c}\right)\left(u_{, \alpha}^{i}-u_{c, \alpha}^{i}\right) \\
& +\frac{\partial \widetilde{G}}{\partial A_{\alpha}^{i}}\left(\nabla \mathbf{u}_{c}, \operatorname{Adj} \nabla \mathbf{u}_{c}, \operatorname{det} \nabla \mathbf{u}_{c}\right)\left((\operatorname{Adj} \nabla \mathbf{u})_{i}^{\alpha}-\left(\operatorname{Adj} \nabla \mathbf{u}_{c}\right)_{i}^{\alpha}\right) \\
& +\frac{\partial G}{\partial d}\left(\nabla \mathbf{u}_{c}, \operatorname{Adj} \nabla \mathbf{u}_{c}, \operatorname{det} \nabla \mathbf{u}_{c}\right)\left(\operatorname{det} \nabla \mathbf{u}-\operatorname{det} \nabla \mathbf{u}_{c}\right) d x,
\end{aligned}
$$

where $\widetilde{G}$ is the function corresponding to $\widetilde{W}$ in (1.5) and the partial derivatives of $\widetilde{G}$ refer to the derivatives of $\widetilde{G}=\widetilde{G}(F, A, d)$ with respect to its arguments. We denote the integral functional on the right-hand side of this inequality by $\widetilde{E}(\mathbf{u})$. It is easily verified that

(i) $E(\mathbf{u}) \geq \widetilde{E}(\mathbf{u})$ and $\mathbf{u} \in W^{1, P}(B)$,

(ii) $\widetilde{E}\left(\mathbf{u}_{c}\right)=E\left(\mathbf{u}_{c}\right)$.

Thus, to prove that $\mathbf{u}_{c}$ is a local minimiser of $E$, it is sufficient to prove that $\mathbf{u}_{c}$ is a local minimiser of $\widetilde{E}$. To analyse the structure of $\widetilde{E}$ we write $\mathbf{u}=\mathbf{u}_{c}+\phi$ where $\phi \in W_{0}^{1, P}(B)$ and observe that $\widetilde{E}$ then takes the form

$$
\begin{aligned}
\widetilde{E}(\mathbf{u})= & E\left(\mathbf{u}_{c}\right)+\int_{B} \frac{\partial W}{\partial F_{\alpha}^{i}}\left(\nabla \mathbf{u}_{c}\right) \phi_{, \alpha}^{i} \\
& +\int_{B} \kappa|\nabla \phi|^{2}+\frac{\partial \widetilde{G}}{\partial A_{\alpha}^{i}}\left(\nabla \mathbf{u}_{c}, \operatorname{Adj} \nabla \mathbf{u}_{c}, \operatorname{det} \nabla \mathbf{u}_{c}\right)(\operatorname{Adj} \nabla \phi)_{i}^{\alpha} \\
& +\frac{\partial \widetilde{G}}{\partial d}\left(\nabla \mathbf{u}_{c}, \operatorname{Adj} \nabla \mathbf{u}_{c}, \operatorname{det} \nabla \mathbf{u}_{c}\right)\left[\operatorname{det} \nabla \phi+(\operatorname{Adj} \nabla \phi)_{i}^{\alpha} u_{c, \alpha}^{i}\right] .
\end{aligned}
$$

Notice that the linear terms in $\nabla \phi$ in the above expression are a weak form of the Euler-Lagrange equations (1.2). Thus to prove that $\mathbf{u}_{c}$ is a minimiser of $E$ it is sufficient to prove that $\mathbf{u}_{c}$ is such a weak solution and that $\widetilde{\mathscr{F}}(\phi)$ is nonnegative for admissible variations $\phi$, where

$$
\begin{aligned}
\widetilde{\mathscr{F}}(\phi) \stackrel{\text { def }}{=} & \int_{B} \kappa|\nabla \phi|^{2}+\frac{\partial \widetilde{G}}{\partial A_{\alpha}^{i}}\left(\nabla \mathbf{u}_{c}, \operatorname{Adj} \nabla \mathbf{u}_{c}, \operatorname{det} \nabla \mathbf{u}_{c}\right)(\operatorname{Adj} \nabla \phi)_{i}^{\alpha} \\
& +\frac{\partial \widetilde{G}}{\partial d}\left(\nabla \mathbf{u}_{c}, \operatorname{Adj} \nabla \mathbf{u}_{c}, \operatorname{det} \nabla \mathbf{u}_{c}\right)\left[\operatorname{det} \nabla \phi+(\operatorname{Adj} \nabla \phi)_{i}^{\alpha} u_{c, \alpha}^{i}\right] .
\end{aligned}
$$

We use this approach to prove our stability result for cavitating equilibria. In the next section we gather results on cavitating equilibria which we will require in our analysis.

2. Cavitating equilibria: properties and stability in $W^{1, P}, P>3$. We first consider radial deformations $\mathbf{u}$ of the ball $B$ which are therefore expressible as

$$
\mathbf{u}(\mathbf{x})=\frac{r(R)}{R} \mathbf{x}, \quad \text { where } R=|\mathbf{x}| .
$$


Thus

$$
\nabla \mathbf{u}(\mathbf{x})=\frac{r(R)}{R}\left(1-\frac{\mathbf{x} \otimes \mathbf{x}}{R^{2}}\right)+r^{\prime}(R) \frac{\mathbf{x} \otimes \mathbf{x}}{R^{2}} .
$$

We restrict attention to the displacement boundary-value problem so that

$$
\mathbf{u}(\mathbf{x})=\lambda \mathbf{x} \quad \text { on } \partial B
$$

for some $\lambda>0$. This condition translates via (2.1) to

$$
r(1)=\lambda \text {. }
$$

If the stored energy function $W$ is frame indifferent and isotropic, i.e., $W(F Q)=$ $W(Q F)=W(F)$ for all $Q \in \mathrm{SO}(3), F \in M^{3 \times 3}$ then it is well known (see [24]) that there is a symmetric function $\Phi: \mathbf{R}_{++}^{3} \rightarrow \mathbf{R}$ satisfying $W(F)=\Phi\left(v_{1}, v_{2}, v_{3}\right)$ for all $F \in M^{3 \times 3}$ where the $v_{i}$ 's are the principal stretches which are the singular values of $F$, i.e., the eigenvalues of $\sqrt{F^{\mathrm{T}} F}$. (Here $\mathbf{R}_{++}^{3}$ denotes the positive octant of $\mathbf{R}^{3}$.) For radial deformations (2.1) the singular values of $\nabla \mathbf{u}$ (given by (2.2)) are easily verified to be

$$
v_{1}=r^{\prime}(R), \quad v_{2}=v_{3}=\frac{r(R)}{R} \text {. }
$$

The radial equilibrium equation is the ordinary differential equation

$$
\frac{d}{d R}\left[R^{2} \Phi_{, 1}\left(r^{\prime}, \frac{r}{R}, \frac{r}{R}\right)\right]=2 R \Phi_{, 2}\left(r^{\prime}, \frac{r}{R}, \frac{r}{R}\right) \text {. }
$$

This is the Euler-Lagrange equation for (1.1) when restricted to radial deformations of the form (2.1). The homogeneous deformations $\mathbf{u}_{\mathrm{hom}}(\mathbf{x}) \equiv \lambda \mathbf{x}$ correspond to the radial function $r_{\text {hom }}(R) \equiv \lambda R$ which is always a solution of (2.5) satisfying (2.3).

We say that $\mathbf{u}_{0}(\mathbf{x})=\left(r_{0}(R) / R\right) \mathbf{x}$ is a cavitating equilibrium if $r_{0} \in C^{2}((0,1])$ is a solution of $(2.5)$ on $(0,1)$ satisfying $r_{0}(0)>0, r_{0}^{\prime}(R)>0$ on $(0,1]$ and the natural boundary condition

$$
\lim _{R \rightarrow 0}\left(\frac{R}{r_{0}(R)}\right)^{2} \Phi_{, 1}\left(r_{0}^{\prime}(R), \frac{r_{0}(R)}{R}, \frac{r_{0}(R)}{R}\right)=0 .
$$

Notice that if $r_{0}(0)>0$ then the deformation (2.1) produces a hole of radius $r_{0}(0)$ at the centre of $B$ and (2.6) is the condition that the radial component of the Cauchy stress vanishes on the surface of the cavity.

We suppose henceforth that $\Phi$ is $C^{3}$ on its domain of definition and satisfies the following constitutive hypotheses:

$$
\Phi_{, 11}\left(v_{1}, v_{2}, v_{3}\right)>0 \text { for all } v_{i} \text {. }
$$

(This is the tension-extension inequality; see [24].)

$\frac{v_{i} \Phi_{, i}\left(v_{1}, v_{2}, v_{3}\right)-v_{j} \Phi_{, j}\left(v_{1}, v_{2}, v_{3}\right)}{v_{i}-v_{j}} \geq 0 \quad$ for all $i \neq j, \quad v_{i} \neq v_{j}$

(These are the weakened Baker-Ericksen inequalities; see [24].) 
(H3)

For each $a \in(0, \infty)$ there exist $v, w \in(0, \infty)$ satisfying $\Phi_{, 1}(v, a, a)>0$

$$
\text { and } \Phi_{, 1}(w, a, a)<0 \text {. }
$$

(This condition typically holds, for example, if the stored energy function $W$ satisfies $W(F) \rightarrow \infty$ as $\operatorname{det} F \rightarrow 0$.)

Under the above hypotheses we obtain the following result.

Proposition 2.1. Let $\Phi$ satisfy (H1)-(H3). Then any cavitating equilibrium $r_{0}$ can be extended to $(0, \infty)$ as a solution of $(2.5)$ with

(i) $\frac{r_{0}(R)}{R}>r_{0}^{\prime}(R)>0$ for $R \in(0, \infty)$,

(ii) $\lim _{R \rightarrow \infty} \frac{r_{0}(R)}{R}=\lim _{R \rightarrow \infty} r_{0}^{\prime}(R)=\lambda_{\text {crit }}$ for some $\lambda_{\text {crit }} \in[1, \infty)$,

(iii) $\frac{r_{0}(R)}{R}$ is monotone decreasing in $R$ and $\lim _{R \rightarrow 0} \frac{r_{0}(R)}{R}=\infty$.

Given any $\lambda>\lambda_{\text {crit }}$ there is a unique $c(\lambda)$ such that

$$
\lambda=c(\lambda) r_{0}\left(\frac{1}{c(\lambda)}\right)
$$

and $c(\lambda) \searrow 0$ as $\lambda \searrow \lambda_{\text {crit }}$. Given any other cavitating equilibrium $\tilde{r} \in C^{2}((0,1])$ then $\tilde{r}(R) \equiv c r_{0}\left(\frac{R}{c}\right)$ for some $c \in \mathbf{R}$ (i.e., all other cavitating equilibria are rescalings of this extended solution).

Proof. (i) and (ii) follow from [21, Proposition 1.6] on noting that the proof still holds under our assumption (H3). (iii) follows from (i) since $r_{0}(0)>0$. The final parts of the Proposition follow from (ii), (iii), the invariance of (2.5) under the scaling $(R, r) \rightarrow(\alpha R, \alpha r), \alpha>0$ and the uniqueness result in [21, Theorem 3.8].

REMARK. It follows from the last Proposition that, by taking an appropriate rescaling of $r_{0}$ if necessary, we may always normalise and assume $r_{0}(0)=1$ so that the constant $c$ appearing in the Proposition is the radius of the cavity produced.

REMARK. Under suitable additional hypotheses on $\Phi$ (see $[3,21,22])$ it can be shown that, in the class of radial mappings, the homogeneous map $\mathbf{u}_{\text {hom }}(\mathbf{x}) \equiv \lambda \mathbf{x}$ is the global minimiser of the stored energy functional for all $\lambda \leq \lambda_{\text {crit }}$ and that for $\lambda>\lambda_{\text {crit }}$ a cavitating equilibrium is the global minimiser.

Lemma 2.2. Let $\mathbf{u}_{0}$ be a radial cavitating equilibrium. Then $\mathbf{u}_{0}$ can be extended to $\mathbf{R}^{3} \backslash\{\mathbf{0}\}$ as a solution of (1.2) and

$$
\begin{aligned}
\nabla \mathbf{u}_{0}(\mathbf{x}) & \rightarrow \lambda_{\text {crit }} I & \text { as }|\mathbf{x}| \rightarrow \infty, \\
\operatorname{Adj} \nabla \mathbf{u}_{0}(\mathbf{x}) & \rightarrow \lambda_{\text {crit }}^{2} I & \text { as }|\mathbf{x}| \rightarrow \infty, \\
\operatorname{det} \nabla \mathbf{u}_{0}(\mathbf{x}) & \rightarrow \lambda_{\text {crit }}^{3} & \text { as }|\mathbf{x}| \rightarrow \infty,
\end{aligned}
$$

where $I$ represents the $3 \times 3$ identity matrix.

Proof. This result follows immediately from Proposition 2.1 (ii) and (2.2).

Our next proposition gives a more accurate representation of the asymptotic behaviour of the extended solution.

Proposition 2.3. Let $r_{0} \in C^{2}((0, \infty))$ be a cavitating solution. Then

(i) $\frac{r_{0}(R)}{R}=\lambda_{\text {crit }}+O\left(1 / R^{3}\right)$ as $R \rightarrow \infty$,

(ii) $r_{0}^{\prime}(R)=\lambda_{\text {crit }}+O\left(1 / R^{3}\right)$ as $R \rightarrow \infty$. 
Proof. We make the change of variables

$$
v=\frac{r}{R}, \quad e^{s}=R,
$$

and so

$$
\frac{d v}{d s}=\dot{v}=r^{\prime}-\frac{r}{R},
$$

which gives $(2.5)$ the autonomous form

$$
\frac{d}{d s} \Phi_{, 1}(\dot{v}+v, v, v)=2\left[\Phi_{, 2}(\dot{v}+v, v, v)-\Phi_{, 1}(\dot{v}+v, v, v)\right] .
$$

Hence for any solution $v(s)$

$$
\begin{aligned}
\frac{\ddot{v}(s)}{\dot{v}(s)} & =\frac{d \dot{v}}{d v} \\
& =2\left[\frac{\Phi_{, 2}(\dot{v}+v, v, v)-\Phi_{, 1}(\dot{v}+v, v, v)}{\dot{v} \Phi_{, 11}(\dot{v}+v, v, v)}-\frac{\Phi_{, 12}(\dot{v}+v, v, v)}{\Phi_{, 11}(\dot{v}+v, v, v)}\right]-1 \\
& =2\left[\frac{\int_{0}^{1}\left[\Phi_{, 21}(t \dot{v}+v, v, v)-\Phi_{, 11}(t \dot{v}+v, v, v)\right] d t-\Phi_{, 12}(\dot{v}+v, v, v)}{\Phi_{, 11}(\dot{v}+v, v, v)}\right]-1 \\
& \stackrel{\text { def }}{=} G(v, \dot{v})
\end{aligned}
$$

where $G$ is $C^{1}$ on its domain $H=\left\{(v, \dot{v}) \in \mathbf{R}^{2}: v>0, \dot{v}+v>0\right\}$.

It now follows that $r_{0}$ gives rise to a solution $v(s)$ of $(2.13)$ and hence to a solution $\dot{v}=f(v)$ of (2.14) satisfying $f\left(\lambda_{\text {crit }}\right)=0$. Expanding $f$ in a Taylor series around $\lambda_{\text {crit }}$ gives

$$
f(v)=-3\left(v-\lambda_{\text {crit }}\right)+g\left(v-\lambda_{\text {crit }}\right)
$$

where $\left|\frac{g\left(v-\lambda_{\text {ciil }}\right)}{\left(v-\lambda_{\text {crit }}\right)^{2}}\right| \leq$ constant for $\left|v-\lambda_{\text {crit }}\right|$ sufficiently small. Thus $\dot{v}(s)=f(v(s))=$ $-3\left(v(s)-\lambda_{\text {crit }}\right)+g\left(v(s)-\lambda_{\text {crit }}\right)$ for all $s$. Since $v(s)>\lambda_{\text {crit }}$ for all $s$, standard arguments now imply that $e^{3 s}\left|v(s)-\lambda_{\text {crit }}\right| \leq$ constant for large $s$ and (i) follows from (2.11). (ii) now follows from (2.12), (2.15), and (i).

LemMA 2.4. Let $r_{0} \in C^{2}((0, \infty))$ be a cavitating equilibrium solution. Then

$$
\frac{d}{d R}\left(\operatorname{det} \nabla \mathbf{u}_{0}\right)=\frac{d}{d R}\left(r_{0}^{\prime}\left(\frac{r_{0}}{R}\right)^{2}\right)=O\left(\frac{1}{R^{4}}\right) \text { as } R \rightarrow \infty .
$$

Proof. It follows from the radial equilibrium equation (2.5) that $r_{0}$ satisfies

$$
\begin{aligned}
r_{0}^{\prime \prime}= & \frac{2}{R}\left(\frac{r_{0}}{R}-r_{0}^{\prime}\right) \frac{1}{\Phi_{, 11}\left(r_{0}^{\prime}, \frac{r_{0}}{R}, \frac{r_{0}}{R}\right)} \\
& \times\left[\frac{\Phi_{1}\left(r_{0}^{\prime}, \frac{r_{0}}{R}, \frac{r_{0}}{R}\right)-\Phi_{, 2}\left(r_{0}^{\prime}, \frac{r_{0}}{R}, \frac{r_{0}}{R}\right)}{\left(r_{0}^{\prime}-\frac{r_{0}}{R}\right)}+\Phi_{, 12}\left(r_{0}^{\prime}, \frac{r_{0}}{R}, \frac{r_{0}}{R}\right)\right] .
\end{aligned}
$$

It is now an easy consequence of Proposition 2.1(ii) and Proposition 2.3 that

$$
\left|r_{0}^{\prime \prime}\right|=O\left(\frac{1}{R^{4}}\right) \quad \text { as } R \rightarrow \infty .
$$


The claim of the lemma now follows from (2.16), Proposition 2.3, and Proposition 2.1(ii) on noting that

$$
\frac{d}{d R}\left(r_{0}^{\prime}\left(\frac{r_{0}}{R}\right)^{2}\right)=r_{0}^{\prime \prime}\left(\frac{r_{0}}{R}\right)^{2}+2 r_{0}^{\prime} \frac{r_{0}}{R}\left(r_{0}^{\prime}-\frac{r_{0}}{R}\right) \frac{1}{R} .
$$

We now make the further assumption that the convex function $\widetilde{G}$ corresponding to the polyconvex function $\widetilde{W}$ in (1.6) has the form

$$
\widetilde{G}(F, \operatorname{Adj}, \operatorname{det} F)=\bar{G}(F, \operatorname{Adj} F)+h(\operatorname{det} F),
$$

where $\bar{G}: M^{3 \times 3} \times M^{3 \times 3} \rightarrow \mathbf{R} \cup\{\infty\}$ and $h: \mathbf{R}^{+} \rightarrow \mathbf{R}$ are $C^{3}$ and convex. Before proving our stability result recall that by (2.1), Proposition 2.1 , and the remark following, under our hypotheses if a cavitating equilibrium exists then we may always represent it as

$$
\mathbf{u}_{c}(\mathbf{x})=c \mathbf{u}_{0}\left(\frac{\mathbf{x}}{c}\right)
$$

where $c$ is the radius of the cavity and $\mathbf{u}_{0}$ is a cavitating equilibrium extended as an equilibrium solution to $\mathbf{R}^{3} \backslash\{\boldsymbol{0}\}$. Using this representation our next result demonstrates the stability of $\mathbf{u}_{c}$ for small $c$.

THEOREM 2.5. Let $W$ be given by (1.6), (2.17) and let the corresponding function $\Phi$ of the principal stretches satisfy $\mathrm{H} 1-\mathrm{H} 3$. Let $\mathbf{u}_{c}$ given by (2.18) be a radial cavitating equilibrium. Then given any $\delta \in(0,1)$ and any $M>0$, for all $c$ sufficiently small,

$$
E(\mathbf{u}) \geq E\left(\mathbf{u}_{c}\right) \text { for all }\left(\mathbf{u}-\mathbf{u}_{c}\right) \in W_{0}^{1, P}\left(B \backslash \bar{B}_{\delta}\right), P>3,
$$

with $\left\|\mathbf{u}-\mathbf{u}_{c}\right\|_{\infty} \leq M$.

Proof. As indicated by the arguments in the introduction, to prove (2.19) it is sufficient to prove that (2.19) holds with $E$ replaced by $\widetilde{E}$ (where $\widetilde{E}$ is the functional defined by (1.8)). Since $\mathbf{u}_{c}$ is a smooth solution of (1.2) it follows by a density argument that the linear terms in $\phi$ in (1.8) are zero for all $\left(\mathbf{u}-\mathbf{u}_{c}\right)=\phi \in W_{0}^{1, P}\left(B \backslash \bar{B}_{\delta}\right)$. Thus it is sufficient to prove that $\widetilde{\mathscr{F}}$ defined by (1.9) is nonnegative for all $\phi \in W_{0}^{1, P}\left(B \backslash \bar{B}_{\delta}\right)$ with $\|\phi\|_{\infty} \leq M$, provided $c$ is sufficiently small.

We first express the quadratic part of $\widetilde{\mathscr{F}}$ as

$$
\begin{aligned}
\widetilde{Q}_{c}(\phi)= & \int_{B} \kappa|\nabla \phi|^{2} \\
& +\left[\frac{\partial \widetilde{G}}{\partial A_{\alpha}^{i}}\left(\nabla \mathbf{u}_{c}, \operatorname{Adj} \nabla \mathbf{u}_{c}, \operatorname{det} \nabla \mathbf{u}_{c}\right)-\frac{\partial \widetilde{G}}{\partial A_{\alpha}^{i}}\left(\lambda_{\text {crit }} I, \lambda_{\text {crit }}^{2} I, \lambda_{\text {crit }}^{3}\right)\right](\operatorname{Adj} \nabla \phi)_{i}^{\alpha} \\
+ & {\left[\frac{\partial \widetilde{G}}{\partial d}\left(\nabla \mathbf{u}_{c}, \operatorname{Adj} \nabla \mathbf{u}_{c}, \operatorname{det} \nabla \mathbf{u}_{c}\right) u_{c, i}^{\alpha}\right.} \\
& \left.-\frac{\partial \widetilde{G}}{\partial d}\left(\lambda_{\text {crit }} I, \lambda_{\text {crit }}^{2} I, \lambda_{\text {crit }}^{3}\right) \lambda_{\text {crit }} \delta_{\alpha}^{i}\right](\operatorname{Adj} \nabla \phi)_{i}^{\alpha}
\end{aligned}
$$

for all $\phi$ in $W_{0}^{1, P}\left(B \backslash \bar{B}_{\delta}\right)$. (Here we have used the fact that $\operatorname{Adj} \nabla \phi$ is a null Lagrangian and so the extra terms introduced integrate to zero; see, e.g., [2]). 
Now using (2.18) and Lemma 2.2 we see that

$$
\begin{aligned}
\nabla \mathbf{u}_{c}(\mathbf{x}) & \rightarrow \lambda_{\text {crit }} I, \\
\operatorname{Adj} \nabla \mathbf{u}_{c}(\mathbf{x}) & \rightarrow \lambda_{\text {crit }}^{2} I, \\
\operatorname{det} \nabla \mathbf{u}_{c}(\mathbf{x}) & \rightarrow \lambda_{\text {crit }}^{3},
\end{aligned}
$$

uniformly on $\bar{B} \backslash B_{\delta}$ as $c \rightarrow 0$.

Combining (2.20) and (2.21) we obtain

$$
\widetilde{\mathscr{Q}}_{c}(\phi) \geq \frac{\kappa}{2} \int_{B}|\nabla \phi|^{2} \quad \text { for all } \phi \in W_{0}^{1, P}\left(B \backslash \bar{B}_{\delta}\right)
$$

for all $c$ sufficiently small.

It now remains to estimate the cubic part of $\widetilde{\mathscr{F}}$ : using our assumption $(2.17)$ this is given by

$$
\widetilde{\mathscr{R}}_{c}(\phi)=\int_{B} h^{\prime}\left(\operatorname{det} \nabla \mathbf{u}_{c}\right) \operatorname{det} \nabla \phi=\int_{B} h^{\prime}\left(\rho\left(\frac{R}{c}\right)\right) \operatorname{det} \nabla \phi,
$$

where

$$
\rho\left(\frac{R}{c}\right)=\operatorname{det} \nabla \mathbf{u}_{c}(\mathbf{x})=\operatorname{det} \nabla \mathbf{u}_{0}\left(\frac{\mathbf{x}}{c}\right)=r_{0}^{\prime}\left(\frac{R}{c}\right)\left(\frac{r_{0}\left(\frac{R}{c}\right)}{\frac{R}{c}}\right)^{2}
$$

and $\mathbf{u}_{0}$ is as in (2.18). Integrating (2.23) by parts gives

$$
\begin{aligned}
\widetilde{\mathscr{R}}_{c}(\phi) & =-\int_{B} \frac{1}{3} \phi^{i}(\operatorname{Adj} \nabla \phi)_{i}^{\alpha} \frac{\partial}{\partial x^{\alpha}} h^{\prime}\left(\rho\left(\frac{R}{c}\right)\right) \text { for all } \phi \in W_{0}^{1, P}\left(B \backslash \bar{B}_{\delta}\right) \\
& =-\int_{B} \frac{1}{3} \phi^{i}(\operatorname{Adj} \nabla \phi)_{i}^{\alpha} \frac{x^{\alpha}}{R}\left[h^{\prime \prime}\left(\frac{R}{c}\right) \rho^{\prime}\left(\frac{R}{c}\right) \frac{R}{c}\right] \frac{1}{R} .
\end{aligned}
$$

By the smoothness of $\operatorname{det} \nabla \mathbf{u}_{0}\left(\frac{\mathbf{x}}{c}\right)$ and Lemma 2.2 it follows that $h^{\prime \prime}(\rho(\eta))$ is uniformly bounded for $\eta$ strictly bounded away from zero. Moreover, using Lemma 2.4, we obtain

$$
h^{\prime \prime}(\rho(\eta)) \rho^{\prime}(\eta) \eta \rightarrow 0 \quad \text { as } \eta \rightarrow \infty .
$$

Hence if $0<\delta \leq R$ then $0<\frac{\delta}{c} \leq \frac{R}{c}$ and so

$$
h^{\prime \prime}\left(\rho\left(\frac{R}{c}\right)\right) \rho^{\prime}\left(\frac{R}{c}\right) \frac{R}{c} \rightarrow 0 \text { uniformly on } B \backslash B_{\delta} \text { as } c \rightarrow 0 \text {. }
$$

It now follows from (2.25) and (2.26) that for all $\phi \in W_{0}^{1, P}\left(B \backslash \bar{B}_{\delta}\right)$ with $\|\phi\|_{\infty} \leq M$

$$
\left|\widetilde{\mathscr{R}}_{c}(\phi)\right| \leq \frac{\kappa}{4} \int_{B}|\nabla \phi|^{2}
$$

for all $c$ sufficiently small.

Combining (2.22) and (2.27) we obtain

$$
\widetilde{F}(\phi) \geq \frac{\kappa}{4} \int_{B}|\nabla \phi|^{2} \text { for all } \phi \in W_{0}^{1, P}\left(B \backslash \bar{B}_{\delta}\right),\|\phi\|_{\infty} \leq M,
$$

for all $c$ sufficiently small, completing the proof of the Theorem. 
Corollary 2.6. Given $\bar{M}>0$, there exists $N>0$ such that

$$
E\left(\mathbf{u}_{c}+\phi\right) \geq E\left(\mathbf{u}_{c}\right) \text { for all } \phi \in W_{0}^{1, P}\left(B \backslash \bar{B}_{N c}\right), P>3,
$$

with $\|\phi\|_{\infty}<\bar{M} N c$.

Proof. This follows by slight modification of the arguments in the proof of Theorem 2.5 on noting that the estimate $(2.27)$ for $\widetilde{R}_{c}(\phi)$ still holds under the above assumptions (by (2.25)) for $N$ sufficiently large.

REMARK. The hypotheses of the Corollary imply that the variations $\phi$ are supported outside a "boundary layer" proportional to the cavity radius $c$ and that $\|\phi\|_{\infty}$ is of the order of the thickness of the layer. Finally, we remark that the proofs of Theorem 2.5 and Corollary 2.6 also hold for maps $\phi$ that are constant on $\bar{B}_{\delta}$ and $\bar{B}_{N c}$, respectively (i.e., it is not necessary that the variations $\phi$ vanish on these regions).

3. Stability of cavitating equilibria in $W^{1, P}(B), P<3$. It follows from [3] that the radial cavitating equilibria $\mathbf{u}_{c}$ are in $W^{1, P}(B)$ for $P<3$. We demonstrate that $\mathbf{u}_{c}$ is not an isolated minimiser of the energy in the sense that given any $\varepsilon>0$ there exists $\mathbf{u} \in W^{1, P}(B) \cap L^{\infty}(B)$ with $\left\|\mathbf{u}-\mathbf{u}_{c}\right\|_{\infty}<\varepsilon$ and $E(\mathbf{u})=E\left(\mathbf{u}_{c}\right)$. The proof is based on a scaling argument (see $[5,14])$. We briefly recall the main idea in our context for the benefit of the reader.

Let $\mathbf{u}: B \rightarrow \mathbf{R}^{3}$ be given by $\mathbf{u}(\mathbf{x})=\lambda \mathbf{x}+\phi(\mathbf{x})$ where $\phi \in W_{0}^{1, P}(B)$. Now consider $\mathbf{u}_{\varepsilon}: \varepsilon B \rightarrow \mathbf{R}^{3}$ defined by

$$
\mathbf{u}_{\varepsilon}(\mathbf{x})=\varepsilon \mathbf{u}\left(\frac{\mathbf{x}}{\varepsilon}\right)
$$

Then

$$
\begin{aligned}
\int_{\varepsilon B} W\left(\nabla \mathbf{u}_{\varepsilon}(\mathbf{x})\right) d x & =\int_{\varepsilon B} W\left(\nabla \mathbf{u}\left(\frac{\mathbf{x}}{\varepsilon}\right)\right) d x \\
& =\varepsilon^{3} \int_{B} W(\nabla \mathbf{u}(\mathbf{y})) d \mathbf{y}=\varepsilon^{3} E(\mathbf{u}) .
\end{aligned}
$$

Thus $\mathbf{u}_{\varepsilon}$ is a deformation of the ball of radius $\varepsilon$ with $\varepsilon^{3}$ times as much energy as the original deformation $\mathbf{u}$ of $B$. Now, up to the exclusion of a set of zero measure, let $B=\bigcup_{i=1}^{\infty} B_{\varepsilon_{i}}\left(\mathbf{x}_{i}\right)$ be a disjoint decomposition of $B$ where $B_{\varepsilon_{i}}\left(\mathbf{x}_{i}\right)=$ $\left\{\mathbf{x} \in \mathbf{R}^{3}:\left|\mathbf{x}-\mathbf{x}_{i}\right|<\varepsilon_{i}\right\}$ are disjoint balls. (This is possible by the Vitali Covering Theorem.) Now define a deformation $\tilde{\mathbf{u}}$ of $B$ by

$$
\tilde{\mathbf{u}}(\mathbf{x})=\lambda \mathbf{x}_{i}+\varepsilon_{i} \mathbf{u}\left(\frac{\mathbf{x}-\mathbf{x}_{i}}{\varepsilon_{i}}\right)=\lambda \mathbf{x}+\varepsilon_{i} \phi\left(\frac{\mathbf{x}-\mathbf{x}_{i}}{\varepsilon_{i}}\right) \quad \text { if } \mathbf{x} \in B_{\varepsilon_{i}}\left(\mathbf{x}_{i}\right) .
$$

Then $\tilde{\mathbf{u}} \in W^{1, P}(B),\left.\tilde{\mathbf{u}}\right|_{\partial B}=\lambda \mathbf{x}$ (see [5] for details) and it is easily verified that by (3.1), since translations do not change the stored energy,

$$
E(\tilde{\mathbf{u}})=\int_{B} W(\nabla \tilde{\mathbf{u}}(\mathbf{x})) d x=\sum_{i=1}^{\infty} \varepsilon_{i}^{3} E(\mathbf{u}) .
$$

Since the $\left\{B_{\varepsilon_{i}}\left(\mathbf{x}_{i}\right)\right\}$ fill $B$, it follows that $\frac{4}{3} \pi=\sum_{i=1}^{\infty} \frac{4}{3} \pi \varepsilon_{i}^{3}$ and hence that $E(\mathbf{u})=$ $E(\tilde{\mathbf{u}})$, i.e., both $\mathbf{u}$ and $\tilde{\mathbf{u}}$ have the same energy. Thus, in general, it is possible to 

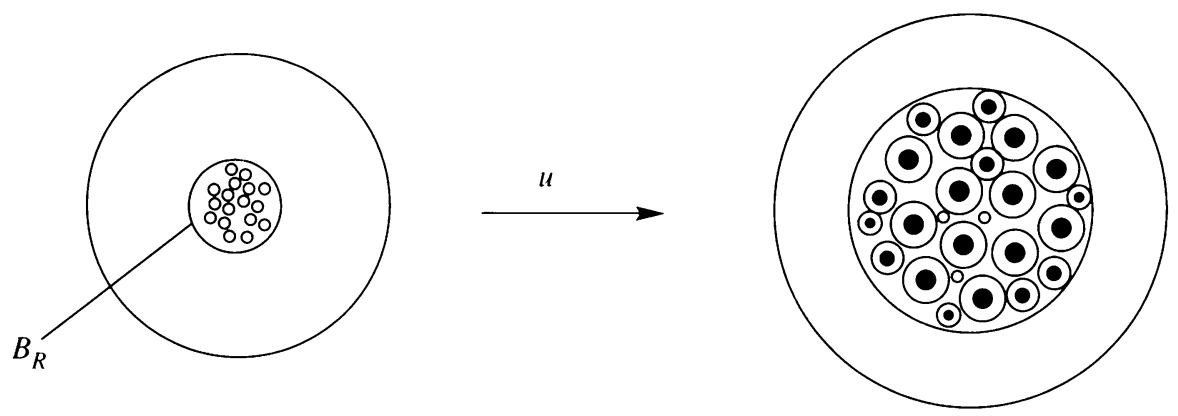

FIG. 1

construct infinitely many distinct maps with the same energy as $\mathbf{u}$ and satisfying the same Dirichlet boundary condition (provided that $\mathbf{u} \not \equiv \lambda \mathbf{x}$ ) .

Now observe that if $\mathbf{u}_{c}(\mathbf{x})=\left(r_{c}(R) / R\right) \mathbf{x}$ is a radial cavitating map then by this construction there are maps producing infinitely many holes with the same energy as $\mathbf{u}_{c}$. Moreover, since the restriction of $\mathbf{u}_{c}$ to any sphere of radius $R \in(0,1)$ is equal to the restriction of an affine map then by the above construction applied to $\mathbf{u}_{c}$ restricted to $B_{R}$ we have deformations of the form given in Fig. 1 with the same energy as $\mathbf{u}_{c}$.

Finally, to obtain maps $\mathbf{u} \in W^{1, P}(B) \cap L^{\infty}(B)$ in any $L^{\infty}$ neighbourhood of $\mathbf{u}_{c}$ with the same energy as $\mathbf{u}_{c}$ we use the above construction to obtain a deformation which produces a thin "shell of holes" together with a cavity at the centre of $B$. Let $0<\varepsilon_{1}<\varepsilon_{2}<1$ and consider the map

$$
\mathbf{u}(\mathbf{x})= \begin{cases}\frac{r_{c}(R)}{R} \mathbf{x} & \text { on } B \backslash B_{\varepsilon_{2}}, \\ \frac{\delta r_{c}(R / \delta)}{R} \mathbf{x} & \text { on } B_{\varepsilon_{1}},\end{cases}
$$

where $\delta=\varepsilon_{1} / \varepsilon_{2}$ so that $\left(\delta / \varepsilon_{1}\right) r_{c}\left(\varepsilon_{1} / \delta\right)=r_{c}\left(\varepsilon_{2}\right) / \varepsilon_{2}$. Then $\mathbf{u}$ is defined on $B \backslash A$, $A=\left\{\mathbf{x} \in \mathbf{R}^{3}: \varepsilon_{1} \leq|\mathbf{x}| \leq \varepsilon_{2}\right\}$ and $\mathbf{u}(\mathbf{x})=\mu_{0} \mathbf{x}$ for all $\mathbf{x} \in \partial A$, where $\mu_{0}=r_{c}\left(\varepsilon_{2}\right) / \varepsilon_{2}$. Extend $\mathbf{u}$ to all of $B$ by defining $\mathbf{u}$ on $A$ by first writing $A$ as the disjoint union of balls of radius $\varepsilon_{2}$ or less and then define a composite map as above in this region using rescalings of $\mathbf{u}_{c}$ restricted to $B_{\varepsilon_{2}}(\mathbf{0})$. The same arguments given above show that $E(\mathbf{u})=E\left(\mathbf{u}_{c}\right)$. Moreover, by choosing $\left|\varepsilon_{2}-\varepsilon_{1}\right|$ sufficiently small we can ensure that $\left\|\mathbf{u}-\mathbf{u}_{c}\right\|_{\infty}$ is as small as required.

4. Stability of cavitating equilibria-variations in cavity shape. In this section we prove stability of cavitating equilibria with respect to a class of deformations which permits (limited) variations in the cavity shape. We use an argument from [19] and an observation of [6].

The class of deformations is defined as follows. Let $\mathbf{u}_{c}$ be an (extended) cavitating radial equilibrium. Then let

$$
\mathbf{u}_{\theta}(\mathbf{x}) \stackrel{\text { def }}{=} \theta(\mathbf{x}) \mathbf{u}_{c}\left(\frac{\mathbf{x}}{\theta(\mathbf{x})}\right)
$$


where $\theta \in C^{1}(\bar{B} \backslash\{\boldsymbol{0}\})$ is positive, bounded, and satisfies

$$
\left.\theta\right|_{\partial B}=1 \text { and } \lim _{t \backslash 0^{+}} \inf \theta(t \mathbf{x})>\delta>0 \text { for any } \mathbf{x} \in B \backslash\{\mathbf{0}\},
$$

for some $\delta>0$.

Clearly the maps $\mathbf{u}_{\theta}$ given by (4.1), (4.2) can produce nonspherical cavities and $\left.\mathbf{u}_{\theta}\right|_{\partial B}=\left.\mathbf{u}_{c}\right|_{\partial B}$ (for example if $\theta$ is of the form $\rho(|\mathbf{x}|) \psi\left(\frac{\mathbf{x}}{|\mathbf{x}|}\right)$ then $\mathbf{u}_{\theta}$ given by (4.1) produces a cavity surface of distance $\rho(\mathbf{0}) \psi\left(\frac{\mathbf{x}}{|\mathbf{x}|}\right) \mathbf{c}$ from the origin in the direction $\mathbf{x})$. Notice also that $\mathbf{u}_{c}$ corresponds to the choice $\theta \equiv 1$ in (4.1). We will prove that $\mathbf{u}_{c}$ is globally minimising in the class of maps given by (4.1), (4.2). We state the result for rank-one convex stored energy functions $W$ (the result then automatically follows for polyconvex stored energy functions since polyconvexity of $W \Rightarrow$ rank-one convexity of $W$, see, e.g., [2]).

THeOREM 4.1. Let the stored energy function $W$ be rank-one convex and let $\mathbf{u}_{c}$ be an (extended) cavitating radial equilibrium. Then

$$
E\left(\mathbf{u}_{\theta}\right) \geq E\left(\mathbf{u}_{c}\right)
$$

for all $\mathbf{u}_{\theta}$ given by (4.1), (4.2).

Proof. It follows from (4.1) that

$$
\nabla \mathbf{u}_{\theta}(\mathbf{x})=\nabla \mathbf{u}_{c}\left(\frac{\mathbf{x}}{\theta}\right)+\left[\mathbf{u}_{c}\left(\frac{\mathbf{x}}{\theta}\right)-\nabla \mathbf{u}_{c}\left(\frac{\mathbf{x}}{\theta}\right) \frac{\mathbf{x}}{\theta}\right] \otimes \nabla \theta .
$$

Since the stored energy function $W$ is rank-one convex it follows from (4.4) that

$$
W\left(\nabla \mathbf{u}_{\theta}(\mathbf{x})\right) \geq W\left(\nabla \mathbf{u}_{c}\left(\frac{\mathbf{x}}{\theta}\right)\right)+\frac{\partial W}{\partial F_{\alpha}^{i}}\left(\nabla \mathbf{u}_{c}\left(\frac{\mathbf{x}}{\theta}\right)\right)\left[u_{c}^{i}\left(\frac{\mathbf{x}}{\theta}\right)-u_{c, \beta}^{i}\left(\frac{\mathbf{x}}{\theta}\right) \frac{x^{\beta}}{\theta}\right] \theta_{, \alpha} .
$$

It follows from [20] that on compact subsets of $\bar{B} \backslash\{\boldsymbol{0}\}$ the right-hand side of (4.5) is a null Lagrangian and from [6] it is equal to the expression

$$
W\left(\nabla \mathbf{u}_{c}(\mathbf{x})\right)+\frac{\partial}{\partial x^{\alpha}}\left[\int_{1}^{\theta(\mathbf{x})} \frac{\partial u^{i}}{\partial t}(\mathbf{x}, t) \frac{\partial W}{\partial F_{\alpha}^{i}}\left(\frac{\partial \mathbf{u}}{\partial \mathbf{x}}(\mathbf{x}, t)\right) d t\right],
$$

where

$$
\mathbf{u}(\mathbf{x}, t)=t \mathbf{u}_{c}\left(\frac{\mathbf{x}}{t}\right) \text {. }
$$

By (4.5), (4.6), (4.2), and the divergence theorem

$$
\begin{aligned}
E\left(\mathbf{u}_{\theta}\right)= & \int_{B} W\left(\nabla \mathbf{u}_{\theta}(\mathbf{x})\right) d x \geq E\left(\mathbf{u}_{c}\right) \\
& +\lim _{\varepsilon \rightarrow 0} \int_{\partial B_{\varepsilon}} \int_{1}^{\theta(\mathbf{x})} \frac{\partial u^{i}}{\partial t}(\mathbf{x}, t) \frac{\partial W}{\partial F_{\alpha}^{i}}\left(\frac{\partial \mathbf{u}}{\partial \mathbf{x}}(\mathbf{x}, t)\right) n^{\alpha} d t d S .
\end{aligned}
$$

We now demonstrate that the integral in (4.8) over $\partial B_{\varepsilon}$ converges to zero as $\varepsilon \rightarrow 0$. To see this notice first that by the isotropy and frame indifference of $W$

$$
\begin{aligned}
\frac{\partial W}{\partial F_{\alpha}^{i}}\left(\frac{\partial \mathbf{u}}{\partial \mathbf{x}}(\mathbf{x}, t)\right)= & \Phi_{, 1}\left(r_{c}^{\prime}\left(\frac{R}{t}\right), \frac{t r_{c}\left(\frac{R}{t}\right)}{R}, \frac{\operatorname{tr}_{c}\left(\frac{R}{t}\right)}{R}\right) \frac{x^{i} x^{\alpha}}{R^{2}} \\
& +\Phi_{, 2}\left(r_{c}^{\prime}\left(\frac{R}{t}\right), \frac{t r_{c}\left(\frac{R}{t}\right)}{R}, \frac{\operatorname{tr}\left(\frac{R}{t}\right)}{R}\right)\left(\delta_{\alpha}^{i}-\frac{x^{i} x^{\alpha}}{R^{2}}\right)
\end{aligned}
$$


(see, e.g., [3]). An easy calculation from (4.7), using the representation $\mathbf{u}_{c}(\mathbf{x})=\frac{r_{c}(R)}{R} \mathbf{x}$, gives

$$
\frac{\partial u^{i}}{\partial t}(\mathbf{x}, t)=\left(\frac{\operatorname{tr}_{c}\left(\frac{R}{t}\right)}{R}-r_{c}^{\prime}\left(\frac{R}{t}\right)\right) \frac{x^{i}}{t} .
$$

Using (4.9) and (4.10), the integral over $\partial B_{\varepsilon}$ in (4.8) then takes the form

$$
\int_{\partial B_{\varepsilon}} \int_{1}^{\theta(\mathbf{x})}\left(r_{c}^{\prime}\left(\frac{R}{t}\right)-\frac{\operatorname{tr}_{c}\left(\frac{R}{t}\right)}{R}\right) \frac{x^{\alpha}}{t} \Phi, 1\left(r_{c}^{\prime}\left(\frac{R}{t}\right), \frac{\operatorname{tr}_{c}\left(\frac{R}{t}\right)}{R}, \frac{\operatorname{tr}_{c}\left(\frac{R}{t}\right)}{R}\right) d t d S .
$$

To estimate (4.11) first note that by Proposition 2.1(i)

$$
\left|\left[\frac{\operatorname{tr}_{c}\left(\frac{R}{t}\right)}{R}-r_{c}^{\prime}\left(\frac{R}{t}\right)\right] \frac{x^{\alpha}}{t}\right| \leq 2 r_{c}\left(\frac{R}{t}\right) .
$$

Hence, since $R=\varepsilon$ on $\partial B_{\varepsilon}$, it follows that (4.11) is bounded by

$$
\int_{\partial B_{\varepsilon}} \int_{1}^{\theta(\mathbf{x})}\left(\frac{t}{\varepsilon}\right)^{2}\left[\frac{\left(\frac{\varepsilon}{t}\right)^{2}}{r_{c}^{2}\left(\frac{\varepsilon}{t}\right)} \Phi_{, 1}\left(r_{c}^{\prime}\left(\frac{\varepsilon}{t}\right), \frac{\operatorname{tr}_{c}\left(\frac{\varepsilon}{t}\right)}{R}, \frac{t r_{c}\left(\frac{\varepsilon}{t}\right)}{\varepsilon}\right)\right] 2 r_{c}^{3}\left(\frac{\varepsilon}{t}\right) d t d S .
$$

It now follows from (4.2) that the arguments in the functions appearing in (4.11) satisfy $\eta=\frac{\varepsilon}{t} \rightarrow 0$ as $\varepsilon \rightarrow 0$ on the domain of integration. Finally, since

$$
\left(\frac{\eta}{r_{c}(\eta)}\right)^{2} \Phi_{, 1}\left(r_{c}^{\prime}(\eta), \frac{r_{c}(\eta)}{\eta}, \frac{r_{c}(\eta)}{\eta}\right) \rightarrow 0 \text { as } \eta \rightarrow 0
$$

by (2.6), this fact combined with the bound (4.13) and the boundedness of $\theta$ imply that the integral in (4.11) converges to zero as $\varepsilon \rightarrow 0$ completing the proof of the result.

Acknowledgments. I would like to thank Charles Stuart and François Meynard for interesting and helpful discussions relating to this work.

\section{REFERENCES}

[1] S. S. Antman and P.V. Negron-Marrero, The remarkable nature of radially symmetric equilibrium states of aelotropic nonlinearly elastic bodies, J. Elasticity 18, 131-164 (1987)

[2] J. M. Ball, Constitutive inequalities and existence theorems in nonlinear elastostatics, Nonlinear Analysis and Mechanics: Heriot-Watt Symposium, Vol. 1 (R. J. Knops, ed.), Pitman, London, 1977, pp. 187-241

[3] J. M. Ball, Discontinuous equilibrium solutions and cavitation in nonlinear elasticity, Philos. Trans. Roy. Soc. London Ser. A 306, 557-611 (1982)

[4] J. M. Ball, J. C. Currie, and P. J. Olver, Null Lagrangians, weak continuity and variational problems of arbitrary order, J. Funct. Anal. 41, 135-174 (1981)

[5] J. M. Ball and F. Murat, $W^{1, P}$-Quasiconvexity and variational problems for multiple integrals, J. Funct. Anal. 58, 225-253 (1984)

[6] J. M. Ball and F. Murat, Remarks on rank-one convexity and quasiconvexity, Ordinary and Partial Differential Equations, Vol. III, Dundee, 1990; Pitman Research Notes Ser. 254, 25-37 (1991)

[7] P. G. Ciarlet, Mathematical Elasticity, Vol. 1: Three-Dimensional Elasticity, North Holland, Amsterdam, 1988

[8] C. O. Horgan, Void nucleation and growth for compressible nonlinearly elastic materials: an example, Internat. J. Solids Structures 29, 279-291 (1992)

[9] C. O. Horgan and R. Abeyaratne, A bifurcation problem for a compressible nonlinearly elastic medium: growth of a microvoid, J. Elasticity 16, 189-200 (1986) 
[10] C. O. Horgan and T. J. Pence, Cavity formation at the center of a composite incompressible nonlinearly elastic sphere, J. Appl. Mech. 56, 302-308 (1989)

[11] C. O. Horgan and T. J. Pence, Void nucleation in tensile loading of a composite incompressible nonlinearly elastic sphere, J. Elasticity 21, 61-82 (1989)

[12] R. D. James and S. J. Spector, The formation of filamentary voids in solids, J. Mech. Phys. Sol. 39, 783-813 (1991)

[13] R. D. James and S. J. Spector, Remarks on $W^{1, p}$-quasiconvexity, interpenetration of matter, and function spaces for elasticity, Ann. Inst. H. Poincaré-Anal. nonlinéaire 9, 263-280 (1992)

[14] C. B. Morrey, Multiple Integrals in the Calculus of Variations, Springer-Verlag, Berlin, 1965

[15] F. Meynard, Cavitation dans un milieu hyperelastique, Thése de Doctorat, École Polytechnique Federale de Lausanne, 1990

[16] F. Meynard, Existence and nonexistence results on the radially symmetric cavitation problem, Quart. Appl. Math. 50, 201-226 (1992)

[17] K. A. Pericak-Spector and S. J. Spector, Nonuniqueness for a hyperbolic system: cavitation in nonlinear elastostatics, Arch. Rational Mech. Anal. 101, 293-317 (1988)

[18] J. Sivaloganathan, Cavitation, the incompressible limit, and material inhomogeneity, Quart. Appl. Math. 49, 521-541 (1991)

[19] J. Sivaloganathan, The generalised Hamilton-Jacobi inequality and the stability of equilibria in nonlinear elasticity, Arch. Rational Mech. Anal. 107, 347-369 (1989)

[20] J. Sivaloganathan, Implications of rank-one convexity, Ann. Inst. H. Poincaré Anal. Non Linéaire 5, 99-118 (1988)

[21] J. Sivaloganathan, Uniqueness of regular and singular equilibria for spherically symmetric problems of nonlinear elasticity, Arch. Rational Mech. Anal. 96, 96-136 (1986)

[22] J. Sivaloganathan, A field theory approach to stability of equilibria in radial elasticity, Math. Proc. Cambridge Philos. Soc. 99, 589-604 (1986)

[23] C. A. Stuart, Radially symmetric cavitation for hyperelastic materials, Ann. Inst. H. Poincaré Anal. Non Linéaire 2, 33-66 (1985)

[24] C. Truesdell and W. Noll, The non-linear field theories of mechanics, Handbuch der Physik III/3, Springer-Verlag, Berlin, 1965 